\title{
R-spondin 1 deficiency in mice improves glycaemic control in association with increased beta cell mass
}

\author{
V. S. C. Wong • A. H. Oh • A. A. Chassot • \\ M. C. Chaboissier $\cdot$ P. L. Brubaker
}

Received: 22 November 2010 / Accepted: 10 March 2011 /Published online: 12 April 2011

(C) Springer-Verlag 2011

\begin{abstract}
Aims/hypothesis Roof plate-specific spondin (R-spondin1; RSPO1) is a modulator of canonical Wg (wingless) plus Int1 (chromosomal integration site of mouse mammary tumour virus on mouse chromosome 15) (cWNT) signalling that induces cWNT target genes. We have demonstrated that Rspol is expressed in murine beta cells, and that it stimulates proliferation and insulin secretion, and inhibits cytokine-induced apoptosis, in mouse insulinoma (MIN6) and beta cells. We thus investigated the role of RSPO1 in beta cells in vivo using Rspol $^{-1-}$ mice.

Methods The effects of Rspol deficiency were assessed by determination of cWNT signalling, glucose tolerance and beta cell mass.

Results Rspol ${ }^{-1-}$ mice demonstrated an $82 \%$ reduction in RSPO1 transcripts and a $61 \%$ reduction in the signal detected by an RSPO1 antibody, as well as a $47 \%$ decrease in islet cWNT signalling. Despite no differences in body and pancreatic weights or in fasting glycaemia and insulinaemia compared with Rspol $^{+/+}$mice, Rspol ${ }^{-/}$animals had improved glycaemic control after oral glucose challenge $(p<0.05)$, with no difference in insulin sensitivity, but an
\end{abstract}

V. S. C. Wong · A. H. Oh · P. L. Brubaker $(\bowtie)$

Department of Physiology,

Room 3366 Medical Sciences Building, University of Toronto,

1 King's College Circle,

Toronto, ON, Canada M5S 1A8

e-mail: p.brubaker@utoronto.ca

P. L. Brubaker

Department of Medicine, University of Toronto,

Toronto, ON, Canada

\section{A. A. Chassot • M. C. Chaboissier}

Inserm, Université de Nice-Sophia Antipolis,

Laboratoire de Génétique du Développement Normal et Pathologique,

Nice, France enhanced insulin response over $30 \mathrm{~min}(p<0.05)$; glucagon responses were normal. Rspol deficiency also resulted in a twofold increase in beta cell mass $(p<0.05)$ in association with 2- and 12-fold increases in the number of beta cells positive for antigen identified by monoclonal antibody Ki67 (Ki67) $(p<0.01)$ and insulin-positive ductal cells $(p<0.05)$, respectively. No change in the number of TUNEL-positive beta cells was detected. Islets isolated from Rspol $^{--}$animals displayed no differences in glucose-induced insulin secretion or in glucose suppression of glucagon.

Conclusions/interpretation The present study reveals an unexpected role for RSPO1 as a regulator of both beta cell proliferation and neogenesis in vivo, and reinforces the importance of cWNT signalling for the maintenance of normal pancreatic beta cell behaviour.

Keywords Apoptosis · Beta cells · Diabetes · Glucagon · Insulin · Neogenesis · Proliferation · R-spondin $1 \cdot$ Secretion WNT signalling
Abbreviations
cWNT Canonical WNT
FRZ Frizzled
GSIS Glucose-stimulated insulin secretion
GSK3 Glycogen synthase kinase 3
HRP Horseradish peroxidase
Ig Immunoglobulin
ITT Insulin tolerance test
Ki67 Antigen identified by monoclonal antibody Ki67
LRP Lipoprotein receptor-related protein
RSPO Roof-plate spondin
TCF4 T cell factor 4
WNT Wg (wingless) plus Int1 (chromosomal integration site of mouse mammary tumour virus on mouse chromosome 15) 


\section{Introduction}

Type 2 diabetes is a complex metabolic disorder that is increasingly being associated with a loss of functional beta cell mass [1]. Thus, there is a continual growth in the number of studies focused on strategies to maintain and/or promote beta cell growth and function. Interestingly, the canonical Wg (wingless) plus Int1 (chromosomal integration site of mouse mammary tumour virus on mouse chromosome 15) (canonical WNT [cWNT]) signalling pathway has recently been implicated in the growth as well as the function of beta cells. In most cells of the body, activation of this pathway by WNT-ligand binding to the frizzled (FRZ)/lipoprotein receptor-related protein (LRP) co-receptors results in inhibition of a degradation complex consisting of adenopolyposis coli, glycogen synthase kinase 3 (GSK3)- $\beta$ and axin, leading to liberation of the transcriptional co-activator, $\beta$-catenin [2]. Initial studies identified multiple WNT ligands, FRZ receptors and other modulators of the cWNT signalling pathway in the developing and mature murine pancreas [3-11]. However, further analyses revealed that the cWNT pathway is also involved in regulating mature beta cell growth. Hence, it has been demonstrated that overproduction of constitutively active $\beta$-catenin in the murine beta cell leads to activation of cWNT signalling and a concomitant increase in beta cell proliferation and total mass [12]. In contrast, ectopic production of negative regulators of cWNT signalling, such as AXIN or GSK3- $\beta$, decreases beta cell proliferation as well as mass $[12,13]$. Moreover, cWNT signalling is also implicated in regulating beta cell function independent of growth. Studies using $\mathrm{Lrp5}^{-1-}$ mice revealed an impairment in glucose tolerance due to reduced glucose-stimulated insulin secretion (GSIS) and this occurred in association with a significant decrease in islet mRNA levels of beta cell transcription factors (e.g. Tcf1 [also known as $V p s 72$ ], $T c f 2$ [also known as Hnflb], Foxal, Hnf4a [also known as $H n f 4 a]$ ), glucokinase and insulin-signalling proteins [14]. In line with this observation, incubation of primary mouse islets and INS1 cells in vitro with adipocyte-derived WNT molecules increases insulin secretion and transcription of glucokinase [15].

The roof plate-specific spondin (R-spondin; RSPO) protein family consists of four related members (RSPO1-4) that have structural similarities with conserved cysteinerich furin-like and thrombospondin domains [16]. Numerous studies have demonstrated that RSPO1 is a regulator of the cWNT signalling pathway, both in development [17-19] and in the adult mouse [17]. Although the precise mechanism by which RSPO1 activates cWNT signalling remains unclear, several recent studies have demonstrated that RSPO family members function as ligands and/or modulators of the FRZ/LRP co- receptors $[20,21]$. As a result of these interactions, RSPO induces the cWNT signalling pathway and initiates WNT target gene expression [20].

We have recently demonstrated the presence of Rspol mRNA transcripts in murine pancreas and isolated islets, as well as in the mouse insulinoma (MIN6) and murine beta tumour cell $(\beta-\mathrm{TC})$ beta cell lines [11]. We have further used these in vitro models to establish that RSPO1 is a beta cell growth factor, stimulating beta cell proliferation and inhibiting beta cell cytokine-induced apoptosis [11]. RSPO1 also enhances insulin secretion in a glucoseindependent fashion in these cells [11]. However, although RSPO1 has been recently demonstrated to play a role in reproductive development [22], its effects on glucose metabolism in vivo have not been explored. We have therefore now examined whole body glucose homeostasis and markers of beta cell growth and function using the Rspol $^{-/-}$mouse.

\section{Methods}

Animals Rspol ${ }^{-/-}$mice were generated by insertion of the $L a c Z$ gene followed by a neomycin resistance cassette into exon 3 of Rspo1 and were genotyped as previously described by Chassot et al. [22]. Unless otherwise indicated, animals were given ad libitum access to water and standard rodent chow with a $12 \mathrm{~h}$ light/dark cycle. All animal protocols were approved by the Animal Care Committee of the University of Toronto (ON, Canada) and by the Université de NiceSophia Antipolis (France), and all in vivo experiments were performed using mice at both sites (Nice, France and Toronto, Canada). The Rspol ${ }^{-1-}$ mice housed in France were on a C57Bl/6 and 129 mixed strain, while the Toronto Rspol $^{-/-}$mice were back-crossed onto a CD1 background. Preliminary comparison between the two strains showed no differences in body weights, metabolic responses and beta cell mass; therefore, data are shown as combined data from the two colonies. In vitro studies were performed using mice from the Toronto colony only. Wild-type $\left(R_{s p o l}{ }^{+/+}\right)$and Rspol $^{-/-}$mice were age- (6-12 weeks) and sex-matchedno differences between males and females were detected for any of the variables studied.

Metabolic tests Mice were fasted overnight or for $6 \mathrm{~h}$ for OGTTs and insulin tolerance tests (ITTs), respectively. Basal blood samples were collected from a tail vein at $t=0 \mathrm{~min}$ for measurement of glucose using the One Touch Basic glucose meter (a kind gift from Lifescan Canada, Burnaby, BC, Canada). For OGTT, mice were gavaged with glucose $(1.5 \mathrm{mg} / \mathrm{g}$ by mouth) and additional blood samples were collected at $t=10,20,30,60,90$ and $120 \mathrm{~min}$ for glucose measurements, and at $t=0$ and $30 \mathrm{~min}$ for 
determination of plasma insulin and glucagon concentrations using an insulin ELISA for small sample volumes (Crystal Chem, Chicago, IL, USA) and a glucagon radioimmunoassay kit (Linco Research, St Louis, MO, USA), respectively. For ITT, mice were injected (i.p.) with human biosynthetic insulin $(0.3 \mathrm{U} / \mathrm{kg}$; Novo Nordisk Pharmaceutical Industries, Toronto, ON, Canada), and additional blood samples were collected at $t=10,20,30$, 60, 90 and 120 min for glucose measurements.

Immunological and morphometric analyses Mouse pancreas was divided into three portions, and the middle section was weighed, fixed in formalin (Sigma-Aldrich, Oakville, ON, Canada), paraffin-embedded and sectioned. Liver, adipose, muscle and jejunal samples were similarly fixed, sectioned and then stained with haematoxylin and eosin for gross morphometric analysis. For determination of beta cell mass, pancreatic sections were incubated overnight at $4^{\circ} \mathrm{C}$ with a guinea pig anti-insulin antibody (Dako Diagnostics, Mississauga, ON, Canada). The sections were then incubated for $1 \mathrm{~h}$ at room temperature with biotinylated antiguinea pig antibody (Vector Laboratories, Burlington, ON, Canada), and subsequently treated for $1 \mathrm{~h}$ with avidinbiotin complex (Vectastain Elite ABC Kit; Vector Laboratories, Burlingame, CA, USA). Slides were stained with 3,3'-diaminobenzidine tetrahydrochloride (Sigma-Aldrich) for $5 \mathrm{~min}$, washed with tap water, and counterstained with haematoxylin. Pancreatic slides were then scanned at the Advanced Optical Microscopy Facility (Princess Margaret Hospital, Toronto, ON, Canada) and beta cell and total pancreatic area per section were measured using Aperio ImageScope software (Aperio Technologies, Vista, CA, USA). Total beta cell mass for each pancreas was determined as the product of the total cross-sectional beta cell area over the total pancreatic area times the weight of the pancreas.

Staining for $\beta$-catenin was performed as modified from previously described methods [23]. Briefly, pancreatic sections were incubated with mouse anti- $\beta$-catenin (BD Biosciences, Mississauga, ON, Canada) followed by immunoperoxidase detection using biotinylated goat antimouse secondary antibody (Vector Laboratories) and streptavidin-horseradish peroxidase (HRP) (Vector Laboratories), with 3,3'-diaminobenzidine tetrahydrochloride (SigmaAldrich) as above. The number of nuclear $\beta$-catenin-positive islet cells was counted for each pancreas.

Immunoblotting Proteins were collected from pancreatic samples and immunoblotted as previously described by Wong et al. [11], using primary antibodies targeted against mouse RSPO1 (goat immunoglobulin [Ig]G, 1:1,000; R\&D Systems, Minneapolis, MN, USA), or pan-actin (rabbit IgG, 1:1,000; Sigma Aldrich), followed by incubation with
HRP-linked anti-goat (1:2,000; Jackson Immunoresearch Laboratories, West Grove, PA, USA) and anti-rabbit (1:2,000; New England Biolabs, Pickering, ON, Canada) secondary antisera and visualisation using electrochemical luminescence (Amersham Pharmacia Biotech, Baie d'Urfe, QC, Canada).

Semi-quantitative real-time- $P C R$ Murine islets were isolated by collagenase digestion and maintained for 2 days, as previously described [11]. Islets were then lysed using the RNeasy Micro Kit, according to the manufacturer's instructions (Qiagen, Mississauga, ON, Canada). Semi-quantitative RT-PCR was performed in a Chromo4 Continuous Fluorescence Detection unit with Opticon Monitor 3 software (BioRad Laboratories, Mississauga, ON, Canada) using Taqman Gene Expression Assays for specific primers (Applied Biosystems, Foster City, CA, USA). All reactions were performed in duplicate, and control reactions were performed without reverse transcriptase enzyme and/or without template. The linearity of amplification of the Taqman primerprobe sets was verified over nine orders of magnitude (data not shown). Ribosomal protein 18S RNA (Hs99999901_sl) was used as the endogenous control for all quantitative analyses of mRNA expression and was not found to change in response to any of the experimental treatments tested (data not shown). Relative quantification of mRNA levels for the cWNT target genes, $c-M y c$ ([also known as Myc] Mm00487803_m1) and cyclin D1 (mM00432359_m1), the beta cell-specific markers, glucokinase (Gck; Mm00439129_m1), Glut2 ([also known as Slc2a2] Mm00446224_m1), insulin 2 (Ins2; Mm00731595_gH), Pdxl (Mm004355565_m1), and the Rspo isoforms, Rspo1 (Mm00507078_g1), Rspo2 (Mm00555790_m1), Rspo3 (Mm00661105_m1) and Rspo4 (Mm00615419_m1), were calculated using the $\Delta \Delta \mathrm{C}_{\mathrm{t}}$ method [24].

In vitro secretion assays Secretion studies using isolated mouse islets were performed as previously described by Li et al. [25]. Briefly, islets were cultured overnight in $20 \mathrm{mmol} / \mathrm{l}$ glucose RPMI1640 (Gibco/Invitrogen, Burlington, ON, Canada) with $10 \% \mathrm{FBS}$ and penicillin/streptomycin. Islets were then washed and incubated with experimental media that consisted of either low $(2 \mathrm{mmol} / \mathrm{l})$ or high glucose (20 mmol/l) RPMI 1640 for $2 \mathrm{~h}$. Media samples were centrifuged at $700 \mathrm{~g}$ at $4^{\circ} \mathrm{C}$ for $1 \mathrm{~min}$ and the supernatant fraction was collected. Samples were then radioimmunoassayed for insulin and glucagon using insulin and glucagon kits from Linco Research. Islet DNA content was determined by spectrophotometry for normalisation of all data.

Statistical analysis All data are expressed as mean \pm SEM. In some experiments, data were $\log _{10}$ transformed to 
normalise variance for statistical analysis. Data were analysed by Student's $t$ test or by one- or two-way ANOVA, followed by appropriate post hoc testing using Statistical Analysis System software (SAS v9.1.3, Cary, NC, USA). Statistical significance was assumed at $p<0.05$.

\section{Results}

Rspo1 $^{-/}$mice have reduced islet cWNT signalling Rspo $1^{-/-}$ mice were phenotypically normal, consistent with previously published results [22]. They shared similar body weights to their wild-type counterparts with no differences in their pancreatic weights (Fig. 1a, b). For exons 4-5 of Rspo1, semi-quantitative real-time-PCR revealed an $82 \%$ reduction $(p<0.05)$ in pancreatic transcript levels in $R s p o 1^{-/}$ knockout mice (Fig. 1c). To confirm these results, pancreatic lysates from $\mathrm{Rspol}^{+/+}$and $\mathrm{Rspol}^{-/-}$animals were also examined by western blot for RSPO1 protein. As shown in Fig. 1d, Rspol ${ }^{+/+}$pancreatic lysates were positive for RSPO1; the molecular mass of this band was approximately $32 \mathrm{kDa}$ as compared with the expected size of $29 \mathrm{kDa}$, although it corresponded in migration to a band observed in the positive control, murine small intestine. This RSPO1 immunoreactivity was reduced by $61 \%$ in pancreases from Rspo $^{-/}$mice $(p<0.05)$. Consistent with these findings, mRNA transcript levels for the cWNT target genes $c-M y c$ and cyclin D1 were reduced by 51 and $56 \%$, respectively, in Rspol $^{-/}$pancreatic lysates (data not shown), while the number of islet nuclear $\beta$-catenin-positive cells was decreased by $47 \%$ (Fig. 1e, $p<0.05$ ). In contrast, analysis of mRNA levels for the other isoforms of Rspo (Rspo2-4) revealed no a

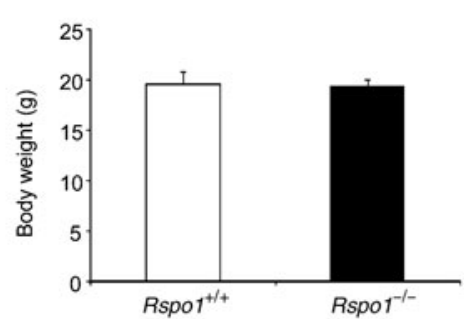

d

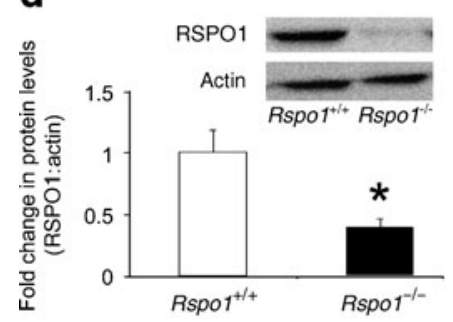

b

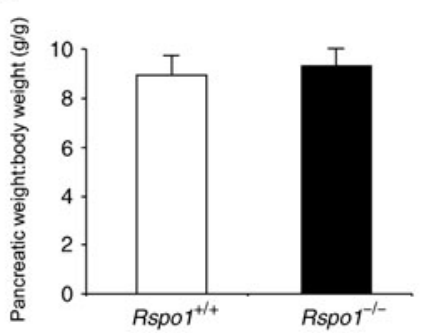

e

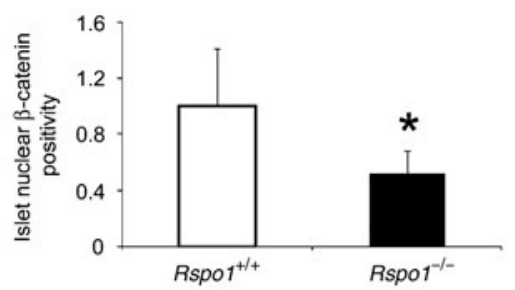

C

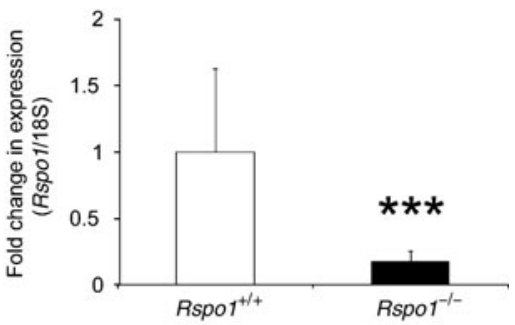

f

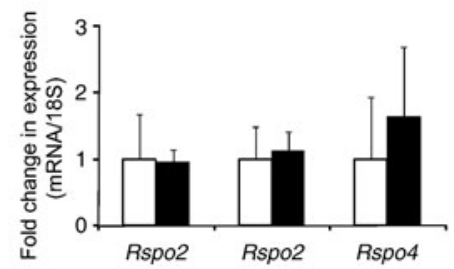

g
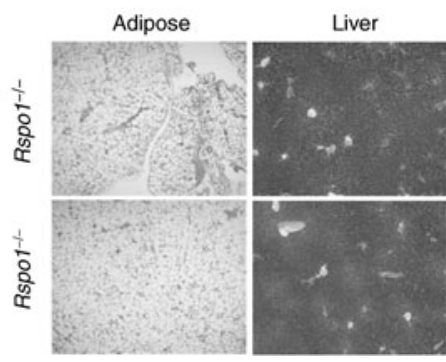

Fig. 1 Rspol $^{-/-}$mice are phenotypically indistinguishable from their wild-type counterparts. a Body weights $(n=11-12)$. b Pancreatic weights normalised to body weights $(n=11-12)$. c Semi-quantitative real-time-PCR analysis of Rspol mRNA in pancreatic lysates. Relative expression levels of Rspol were normalised to 18S rRNA ( $n=4-6)$. d Immunoblotting analysis of RSPO1 from pancreatic lysates. Relative protein levels of RSPO1 were normalised to total actin ( $n=5-6$; representative blot is shown). e Analysis of islet nuclear $\beta$-catenin from Rspol $^{+/+}$and sspol $^{-/-}$mice. The number of islet nuclear $\beta$-catenin-positive cells was normalised to the total number of islet cells per pancreas $(n=8)$. f Semi-quantitative real-time-PCR analysis of Rspo2-4 mRNA in isolated islets of wild-type (white bars) and knockout (black bars) mice. Relative expression levels of Rspo2, -3 and -4 were normalised to $18 \mathrm{~S}$ rRNA expression $(n=4-10)$. g Haematoxylin and eosin stained sections of adipose, liver, skeletal muscle and jejunal tissues $(n=11-12$, representative photomicrographs are shown). ${ }^{*} p<0.05 ; * * * p<0.001$ 
significant changes in $\mathrm{Rspol}^{-/}$compared with wild-type mice, indicating no compensatory responses (Fig. 1f).

Gross morphological analyses of insulin-responsive tissues from wild-type and knockout mice revealed no remarkable changes in adipose tissue, liver or skeletal muscle (Fig. 1g). As RSPO1 is known to be a potent gastrointestinal growth factor [7], we also sought to see if there were changes in the small intestine. Figure $1 \mathrm{~g}$ demonstrates no notable differences between the jejunal architecture of $\mathrm{RspoI}^{+/+}$and $\mathrm{Rspol}^{-/-}$mice.

Rspo $^{-1}$ mice display better glucose handling without changes in insulin sensitivity Fasting glycaemia was in the normal range for wild-type mice $(6.3 \pm 0.5 \mathrm{mmol} / \mathrm{l})$, and was not significantly different in knockout animals (5.2 \pm $0.5 \mathrm{mmol} / \mathrm{l}$; Fig. 2a). However, Rspol $1^{-/}$mice had significantly better glycaemic control after an oral glucose challenge (Fig. 2a). Consistent with this observation, the
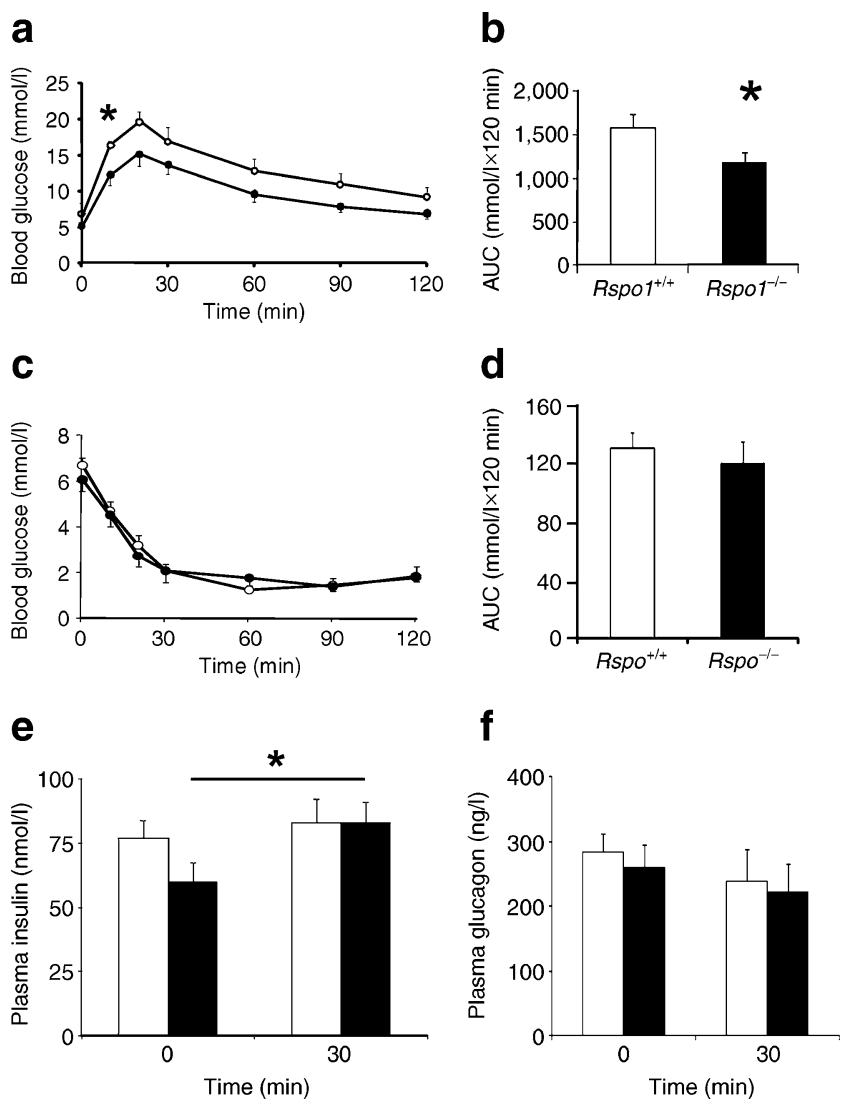

Fig. 2 Rspol $^{-/}$mice have improved glycaemic control. a Glycaemic profiles after an OGTT of wild-type (white circles) and knockout (black circles) mice. b AUC of the glucose excursions at 0 to $120 \mathrm{~min}$ after administration of an oral glucose load $(n=11-12)$. $\mathbf{c}$ Glycaemic responses to an intraperitoneal ITT of wild-type (white circles) and knockout (black circles) mice. d AUC of the glucose excursions at 0 to $120 \mathrm{~min}$ after administration of insulin $(n=11-12)$. Levels of plasma insulin (e) $(n=17-23)$ and glucagon (f) $(n=6-12)$ in wild-type (white bars) and knockout (black bars) mice at $t=0$ and 30 min after administration of an OGTT. $* p<0.05$
AUC for the glycaemic response to oral glucose was significantly lower in knockout animals relative to wildtype mice $(p<0.05$, Fig. $2 b)$. In contrast, the glycaemic response to an insulin tolerance test (Fig. 2c,d), and fasting insulin levels (Fig. 2e) were not altered in knockout animals, suggesting normal insulin sensitivity. However, consistent with the improved glycaemic control in $\mathrm{Rspol}^{-/}$ mice, there was a significant increase in plasma insulin levels at $t=30 \mathrm{~min}$ in response to oral glucose in the Rspol $^{-/}$mice as compared with the muted response observed in the wild-type animals $(p<0.05$, Fig. 2e). Plasma glucagon concentrations were not different between the animals in either the fasting state (e.g. at $t=0 \mathrm{~min}$ ) or at $t=30 \mathrm{~min}$ following the oral glucose challenge (Fig. 2f).

Rspol $^{-/-}$mice have increased beta cell mass, proliferation and neogenesis One possible mechanism underlying the better glycaemic handling in $R s p o I^{-/-}$mice could involve changes in the number of beta cells. Therefore, pancreatic sections were stained for insulin to determine total beta cell mass. Rspo $1^{-/-}$mice were found to have significantly increased beta cell mass, by 2.3 -fold relative to wild-type controls $(p<0.05$, Fig. 3a). To determine the mechanism(s) underlying the changes in beta cell mass, stained sections were first subjected to morphometric analyses. The total number of islets was increased in pancreases from $\mathrm{Rspol}^{-/}$ mice ( $p<0.05$; Fig. $3 b$ ), although islet distribution by size (arbitrarily set as 1, 2-100 or greater than 100 beta cells; Fig. 3b) and average beta cell size (Fig. 3c) were not significantly different between wild-type and knockout animals. However, examination of pancreatic sections that were co-stained for insulin and the proliferative marker, antigen identified by monoclonal antibody Ki67 (Ki67), revealed that proliferation was significantly increased, by 2.3 -fold $(p<0.01)$ in knockout mice relative to wild-type animals, mirroring the changes observed in beta cell mass $(p<0.01$, Fig. 3d). Examination of beta cell apoptosis, detected by insulin and cleaved-caspase 3 co-staining, demonstrated extremely low levels in all pancreases $(\sim 1-2$ apoptotic beta cells per $\sim 1,500$ beta cells), and no differences in beta cell apoptosis could be detected in the $\mathrm{Rspol}^{-/}$ mice (data not shown). However, unexpectedly, there were significantly more insulin-positive ductal cells in the knockout mice (by 12-fold, $p<0.05$ ), an indication of beta cell neogenesis, whereas such cells were almost completely absent in the Rspol $^{+/+}$animals $(p<0.05$, Fig. 3e). Finally, semi-quantitative real-time-PCR demonstrated that Ins2, $P d x l, G c k$ and Glut2 mRNA levels were increased in islets isolated from $\mathrm{Rspol}^{-/}$mice compared with those obtained from wild-type animals $(p<0.05-0.01$, Fig. 3f).

Rspol $^{-/}$mouse islets display normal glucose modulation of hormone secretion Basal insulin release under low 


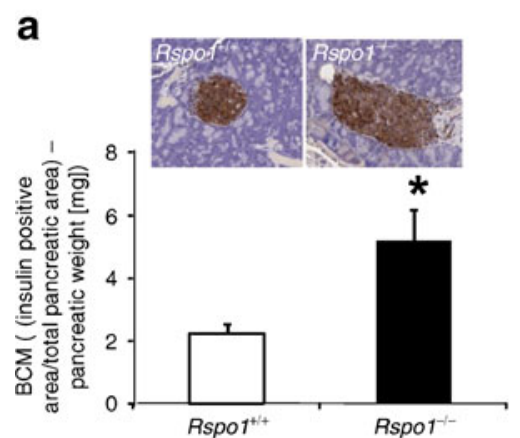

b

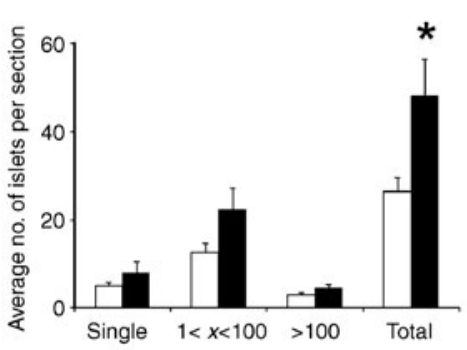

d

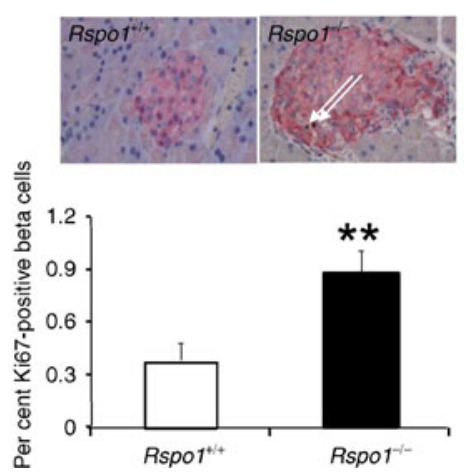

e

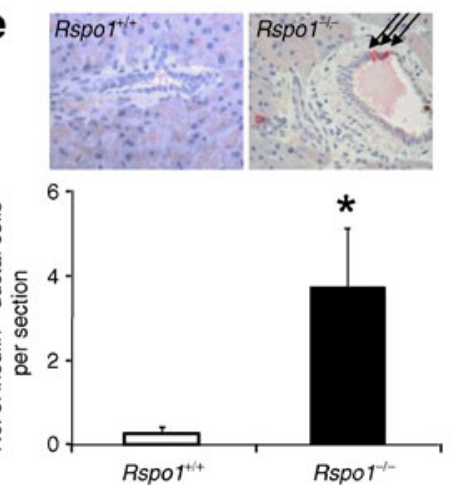

C

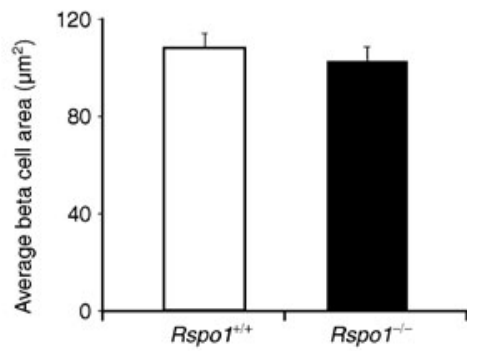

f

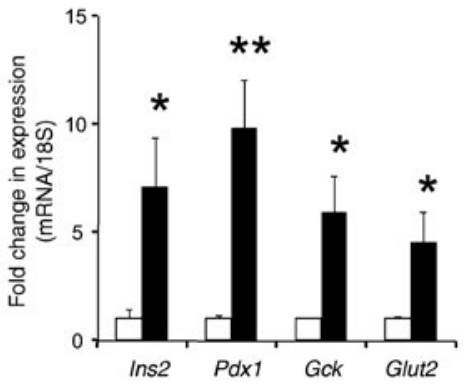

Fig. 3 Rspol $^{-1-}$ mice have increased beta cell mass, proliferation and neogenesis. a Beta cell mass was determined in insulin-stained pancreatic sections from $R s p o I^{+/+}$and $R_{s p o l}{ }^{-/}$mice $(n=11-12)$. Representative islets are shown. b Size distribution and total number of islets in pancreases of Rspol $^{+/+}$(white bars) and Rspol $^{-/}$(black bars) mice. Islets were arbitrarily distributed based on size, as either single insulin-positive cells, between 2 and 100 insulin positive-cells or more than 100 beta cells $(n=11)$. c Average beta cell area $(n=5)$. d Proliferating beta cells as determined by co-staining for insulin and Ki67 ([no. of Ki67 ${ }^{+}$insulin $^{+}$cells $\left.\times 100\right] /$ total number of insulin ${ }^{+}$

glucose conditions was not statistically different in $\mathrm{Rspol}^{-1-}$ mice relative to wild-type controls. Furthermore, incubation of islets with high glucose $(20 \mathrm{mmol} / \mathrm{l})$ for $2 \mathrm{~h}$ stimulated insulin secretion to a similar extent in both groups of animals ( $p=0.055$ in wild-type islets; $p<0.05$ in knockout islets; Fig. 4a). Similarly, glucagon secretion under low and high glucose conditions was not different between $\mathrm{Rspol}^{-/-}$and wild-type islets (Fig. 4b).

\section{Discussion}

RSPO1 has recently been established as a novel regulator of cWNT signalling in the beta cell [11]. Hence, RSPO1 is produced in murine islets as well as in MIN6 and $\beta$-TC beta cells. Furthermore, treatment of MIN6 cells with RSPO1 increases nuclear $\beta$-catenin translocation in association with increased $c-M y c$ and Ins 2 mRNA transcript levels. Finally, the effects of RSPO1 on MIN6 as well as primary murine beta cells in vitro include enhanced cells). The number of proliferating beta cells was normalised to the total number of beta cells and expressed as a percentage (\%). Arrows indicate positive cells $(n=9-10)$. e Beta cell neogenesis as determined by the number of insulin-positive cells in the pancreatic ducts. Arrows indicate positive cells $(n=11)$. f Semi-quantitative real-time-PCR analysis of Ins2, Pdxl, Gck and Glut2 mRNA in isolated islets from Rspol $^{+/+}$(white bars) and Rspol ${ }^{-/}$(black bars) mice $(n=4-10)$. Relative expression levels of each transcript were normalised to $18 \mathrm{~S}$ rRNA expression. ${ }^{*} p<0.05 ;{ }^{*} p<0.01$. BCM, beta cell mass

proliferation and protection from cytokine-induced apoptosis, as well as stimulation of insulin secretion [11]. We now provide novel findings that RSPO1 is also a regulator of whole-body glucose homeostasis via changes in beta cell behaviour in vivo. However, unexpectedly and in contrast
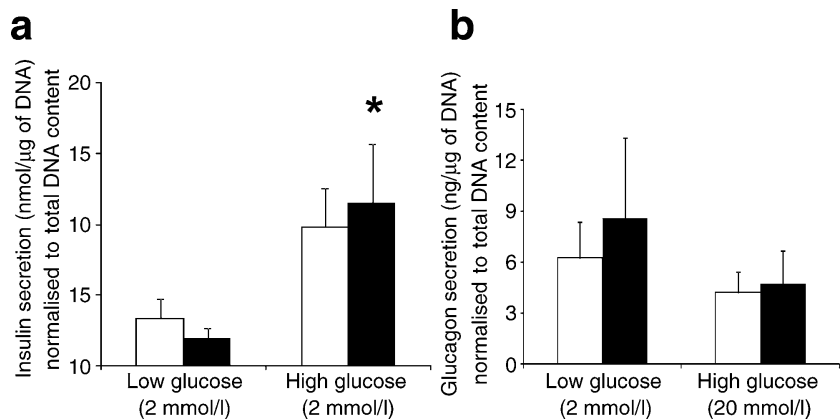

Fig. $4 \mathrm{Rspol}^{-/-}$islets demonstrate normal glucose modulation of hormone secretion. a, b Effects of low $(2 \mathrm{mmol} / \mathrm{l})$ and high $(20 \mathrm{mmol} / \mathrm{l})$ glucose on insulin (a) $(n=10-13)$ and glucagon (b) $(n=8-13)$ from isolated islets of $\mathrm{Rspol}^{+/+}$(white bars) and $\mathrm{Rspol}^{-/}$(black bars) mice, as determined by radioimmunoassay. Results were normalised to total DNA content. $* p<0.05$ 
to our in vitro findings, the effects of Rspol deficiency in vivo resulted in improved glycaemic tolerance in association with enhanced beta cell mass.

In the present study, we confirmed knockout of Rspo1 in the Rspo1-null mice at the level of DNA by genotyping. Previous analysis of these mice by Southern blot also did not reveal a signal [22]. In contrast, very low levels of Rspol mRNA (18\% of normal) were detected in islets from the Toronto $\mathrm{Rspol}^{-/-}$mice by semi-quantitative real-time PCR for exons 4-5, a finding that has recently been confirmed in the colony in France $(10-20 \%$ of normal expression levels; unpublished observations). One possible explanation arises from a recent report of two Rspol transcripts, such that our primers may have detected the second, 'truncated' Rspol transcript (446 bp; transcript ID ENSMUST00000140698). As Rspol was knocked out by replacement of a section of exon3 with a $L a c Z$ gene and a polyA signal, it is also possible that a hybrid transcript is expressed via production of a read-through message - this would result in a protein without the cysteine-rich domain required for RSPO1 interactions with the FRZ/LRP receptors [16]. Thus, although immunoblotting of pancreatic lysates from $\mathrm{Rspol}^{-/-}$mice found detectable but reduced (by 61\%) levels of RSPO1 immunoreactivity, these findings are consistent with the marked decreased in cWNT signalling in these animals, as evidenced by a $>50 \%$ reduction in pancreatic $c-M y c$ and cyclin D1 transcript levels, as well as a $47 \%$ decrease in islet nuclear $\beta$-catenin localisation. Notwithstanding, further studies are clearly required to fully elucidate the identity of the apparent RSPO1 mRNA transcript and immunoreactivity detected in the Rspol $^{-/-}$mice.

Rspol deficiency did not impact overall body and pancreatic weights, and there were no gross morphological differences in three principle insulin-sensitive tissues, namely, adipose tissue, liver and skeletal muscle. Given that RSPO1 is a potent gastrointestinal mitogen $[7,16,26]$, it is interesting to note that there were also no remarkable morphological changes in the small intestine of knockout mice. Together, these findings indicate that, while RSPO1 is essential for normal reproductive system development [22], it may be dispensable for development of these major nutrient handling tissues.

We have previously shown by RT-PCR that Rspo3 and Rspo4, but not Rspo2, are expressed in murine islets [11]. Here we show by the more sensitive technique of semiquantitative real-time-PCR that all three of these isoforms of Rspo are expressed in murine islets. Nonetheless, although previous studies have shown that many transgenic mouse models demonstrated compensatory responses by upregulating other isoforms of the deficient protein [2729], we were unable to detect any upregulation of Rspo2-4 in islets from $\mathrm{Rspol}^{-/-}$mice. This finding suggests that, despite the known similar actions of these four isoproteins in the stimulation of gastrointestinal growth [16], they do not exhibit compensatory actions in the islet.

Somewhat surprisingly, given the stimulatory effect of exogenous RSPO1 on insulin secretion in vitro [11], the glycaemic profile of $\mathrm{Rspol}^{-/-}$mice during an OGTT was found to be significantly reduced relative to $R s p o I^{+/+}$ animals. This phenomenon was associated with a greater change in plasma insulin from 0 to $30 \mathrm{~min}$ in the $R s p o 1^{-/}$ mice, in the absence of any differences in either the glycaemic response to insulin or fasting insulin levels between $\mathrm{RspoI}^{+/+}$and $\mathrm{RspoI}^{-/-}$animals. It is possible that analysis of insulin levels at different time points may reveal further differences between these animals. Nonetheless, these findings suggested that the improved glucose handling in the Rspol-null mice resided at the level of the pancreatic beta cell. Consistent with this possibility, we found a significant increase in beta cell mass in $R_{s p o l^{-1}}$ animals relative to $R s p o I^{+/+}$controls. Notably, this finding was also unexpected given the pro-proliferative and antiapoptotic effects of RSPO1 in the beta cell in vitro [11]. However, such discrepancies in null animal models are not without precedence. For example, although we have previously reported that the incretin hormone glucagonlike peptide-1 enhances beta cell mass in vivo in normal mice and induces beta cell proliferation in vitro, administration of GLP-1 to animals lacking Pi3k $\gamma$ (also known as $P i k 3 c g$ ) causes a paradoxical reduction in both variables [25]. Similarly, despite the known effect of insulin-like growth factor-1 to enhance beta cell proliferation in vitro, beta cell-specific knockout of the insulin-like growth factor-1 receptor does not alter beta cell mass in vivo [30]. Hence, findings made in vitro must often be re-considered in the light of apparently contradictory effects in the more complex in vivo setting.

To examine the mechanisms underlying the increased beta cell mass in $\mathrm{RspoI}^{-/-}$animals in more detail, a variety of factors known to influence the growth of existing beta cells, including proliferation, apoptosis and hypertrophy, were investigated. We found that the increase in beta cell mass occurred in association with an increased number of proliferating beta cells, rather than changes in beta cell apoptosis or beta cell size. However, as the size distribution of the islets was not significantly increased in the knockout animals, these findings suggest that, although significantly increased, the rate of beta cell proliferation was not the major determinant of the increased beta cell mass observed in these mice. Furthermore, the rate of apoptosis in islets from both the wild-type and knockout animals was so low as to effectively preclude a role for this pathway in the regulation of beta cell mass. However, unexpectedly, we did find a profound increase in the number of insulinpositive ductal cells in the $R$ spol ${ }^{-/-}$mouse pancreas. Such 
observations have been characterised by others as beta cell neogenesis, whereby ductal cells differentiate to give rise to new beta cells [31]. Although there are no reports of RSPO1 regulating differentiation in adult tissues, previous studies have implicated the cWNT pathway as an inhibitor of differentiation. Hence, inhibition of the cWNT pathway leads to spontaneous adipogenesis, while stimulation of basal cWNT-mediated signalling prevents pre-adipocyte differentiation [32]. Together, these findings suggest that the ductal cells may be a novel target of RSPO1 action in vivo, with Rspol deficiency in these cells resulting in enhanced beta cell mass through induction of beta cell neogenesis. However, characterisation of this phenomenon is difficult because of issues related to the identification of ductal beta cell progenitors [33, 34]. Future analyses with ductal-specific markers and/or cell-specific knockout animals will be required to determine the precise contribution of ductal transdifferentiation to beta cell mass in the Rspolknockout mouse. Nevertheless, these findings highlight the utility of using global knockout animals to identify novel targets, which would not be possible using either in vitro cell models or cell-specific null animals.

The importance of cWNT signalling in the beta cell has recently been highlighted by reports that single nucleotide polymorphisms in the $T c f 7 l 2$ gene are strongly associated with risk for development of type 2 diabetes [35-39]. Interestingly, $\mathrm{T}$ cell factor 4 (TCF4), the protein product of $T c f 7 l 2$, is a downstream transcription factor that is required for the induction of gene expression by $\beta$-catenin [2]. Although it remains unclear to this date how polymorphisms in $T c f 7 l 2$ translate to functional defects in beta cells, in vitro expression of dominant negative or small interfering RNA knockdown of Tcf7l2 in beta cells or islets decreases both beta cell proliferation and GSIS [9, 10, 40, 41], whereas an overproduction of this transcription factor in mouse and human islets protects beta cells from glucotoxicity- and cytokine-induced apoptosis [10]. Hence, an improved understanding of the role of cWNT signalling in beta cell biology will provide insight into the mechanisms underlying type 2 diabetes risk as well as into the pathophysiology of this disease.

In conclusion, our studies using Rspol ${ }^{-/-}$mice have provided insights as to the role of RSPO1 in the beta cell in vivo, and suggest a novel function for this protein as a negative regulator of beta cell neogenesis. Further studies are clearly warranted if RSPO1 is to be considered as a novel target for the therapeutic treatment of patients with type 2 diabetes.

Acknowledgements The authors are grateful to J. Wysolmerski (Yale University, CT, USA) for the gift of a breeding pair to establish the Toronto colony. This work was supported by an operating grant from the Canadian Diabetes Association (number 2374) and by an equipment grant from the Banting and Best Diabetes Centre (BBDC),
University of Toronto. V. S. C. Wong was supported by a Doctoral Research Award from the Canadian Institutes of Health Research, A. H. Oh by the BBDC Summer Studentship program, A. A. Chassot by a fellowship from the Fondation pour la Recherche Médicale, and P. L. Brubaker by the Canada Research Chairs program.

Duality of interest V. S. C. Wong, A. H. Oh, A. A. Chassot and M. C. Chaboissier declare that there is no duality of interest associated with this manuscript. P. L. Brubaker has received consulting fees from Johnson \& Johnson.

\section{References}

1. Butler AE, Janson J, Bonner-Weir S, Ritzel R, Rizza RA, Butler PC (2003) Beta-cell deficit and increased beta-cell apoptosis in humans with type 2 diabetes. Diabetes 52:102-110

2. MacDonald BT, Tamai K, He X (2009) Wnt/beta-catenin signaling: components, mechanisms, and diseases. Dev Cell 17:9-26

3. Heller RS, Dichmann DS, Jensen J et al (2002) Expression patterns of Wnts, Frizzleds, sFRPs, and misexpression in transgenic mice suggesting a role for Wnts in pancreas and foregut pattern formation. Dev Dyn 225:260-270

4. Heller RS, Klein T, Ling Z et al (2003) Expression of Wnt, Frizzled, sFRP, and DKK genes in adult human pancreas. Gene Expr 11:141-147

5. Pedersen AH, Heller RS (2005) A possible role for the canonical Wnt pathway in endocrine cell development in chicks. Biochem Biophys Res Commun 333:961-968

6. Yi F, Brubaker PL, Jin T (2005) TCF-4 mediates cell type-specific regulation of proglucagon gene expression by beta-catenin and glycogen synthase kinase-3beta. J Biol Chem 280:1457-1464

7. Kim KA, Kakitani M, Zhao J et al (2005) Mitogenic influence of human R-spondin 1 on the intestinal epithelium. Science 309:1256-1259

8. Wang QM, Zhang Y, Yang KM, Zhou HY, Yang HJ (2006) Wnt/ beta-catenin signaling pathway is active in pancreatic development of rat embryo. World J Gastroenterol 12:2615-2619

9. Liu Z, Habener JF (2008) Glucagon-like peptide-1 activation of TCF7L2-dependent Wnt signaling enhances pancreatic beta cell proliferation. J Biol Chem 283:8723-8735

10. Shu L, Sauter NS, Schulthess FT, Matveyenko AV, Oberholzer J, Maedler K (2008) Transcription factor 7-like 2 regulates beta-cell survival and function in human pancreatic islets. Diabetes 57:645653

11. Wong VS, Yeung A, Schultz W, Brubaker PL (2010) R-spondin-1 is a novel beta-cell growth factor and insulin secretagogue. J Biol Chem 285:21292-21302

12. Rulifson IC, Karnik SK, Heiser PW et al (2007) Wnt signaling regulates pancreatic beta cell proliferation. Proc Natl Acad Sci USA 104:6247-6252

13. Liu Z, Tanabe K, Bernal-Mizrachi E, Permutt MA (2008) Mice with beta cell overexpression of glycogen synthase kinase-3beta have reduced beta cell mass and proliferation. Diabetologia 51:623-631

14. Fujino T, Asaba H, Kang MJ et al (2003) Low-density lipoprotein receptor-related protein 5 (LRP5) is essential for normal cholesterol metabolism and glucose-induced insulin secretion. Proc Natl Acad Sci USA 100:229-234

15. Schinner S, Ulgen F, Papewalis C et al (2008) Regulation of insulin secretion, glucokinase gene transcription and beta cell proliferation by adipocyte-derived Wnt signalling molecules. Diabetologia 51:147-154

16. Kim KA, Zhao J, Andarmani S et al (2006) R-spondin proteins: a novel link to beta-catenin activation. Cell Cycle 5:23-26 
17. Kamata T, Katsube K, Michikawa M, Yamada M, Takada S, Mizusawa H (2004) R-spondin, a novel gene with thrombospondin type 1 domain, was expressed in the dorsal neural tube and affected in Wnts mutants. Biochim Biophys Acta 1676:51-62

18. Nam JS, Turcotte TJ, Yoon JK (2007) Dynamic expression of Rspondin family genes in mouse development. Gene Expr Patterns 7:306-312

19. Kazanskaya O, Glinka A, del Barco Barrantes I, Stannek P, Niehrs C, Wu W (2004) R-spondin2 is a secreted activator of Wnt/betacatenin signaling and is required for Xenopus myogenesis. Dev Cell 7:525-534

20. Wei Q, Yokota C, Semenov MV, Doble B, Woodgett J, He X (2007) R-spondin 1 is a high affinity ligand for LRP6 and induces LRP6 phosphorylation and beta-catenin signaling. J Biol Chem 282:15903-15911

21. Binnerts ME, Kim KA, Bright JM et al (2007) R-spondin1 regulates Wnt signaling by inhibiting internalization of LRP6. Proc Natl Acad Sci USA 104:14700-14705

22. Chassot AA, Ranc F, Gregoire EP et al (2008) Activation of betacatenin signaling by Rspo1 controls differentiation of the mammalian ovary. Hum Mol Genet 17:1264-1277

23. Dube PE, Rowland KJ, Brubaker PL (2008) Glucagon-like peptide-2 activates beta-catenin signaling in the mouse intestinal crypt: role of insulin-like growth factor-I. Endocrinology 149:291-301

24. Pfaffl MW (2001) A new mathematical model for relative quantification in real-time RT-PCR. Nucleic Acids Res 29:e45

25. Li LX, MacDonald PE, Ahn DS, Oudit GY, Backx PH, Brubaker PL (2006) Role of phosphatidylinositol 3-kinasegamma in the beta-cell: interactions with glucagon-like peptide-1. Endocrinology 147:3318-3325

26. Dube PE, Forse CL, Bahrami J, Brubaker PL (2006) The essential role of insulin-like growth factor-1 in the intestinal tropic effects of glucagon-like peptide-2 in mice. Gastroenterology 131:589-605

27. Mao C, Tili EG, Dose M et al (2007) Unequal contribution of Akt isoforms in the double-negative to double-positive thymocyte transition. J Immunol 178:5443-5453

28. Terauchi Y, Tsuji Y, Satoh S et al (1999) Increased insulin sensitivity and hypoglycaemia in mice lacking the p85 alpha subunit of phosphoinositide 3-kinase. Nat Genet 21:230-235
29. Mashimo H, Goyal RK (1999) Lessons from genetically engineered animal models. IV. Nitric oxide synthase gene knockout mice. Am J Physiol 277:G745-G750

30. Liu JL (2007) Does IGF-I stimulate pancreatic islet cell growth? Cell Biochem Biophys 48:115-125

31. Juhl K, Bonner-Weir S, Sharma A (2010) Regenerating pancreatic beta-cells: plasticity of adult pancreatic cells and the feasibility of in-vivo neogenesis. Curr Opin Organ Transplant 15:79-85

32. Bennett CN, Ross SE, Longo KA et al (2002) Regulation of Wnt signaling during adipogenesis. J Biol Chem 277:30998-31004

33. Inada A, Nienaber C, Katsuta $H$ et al (2008) Carbonic anhydrase II-positive pancreatic cells are progenitors for both endocrine and exocrine pancreas after birth. Proc Natl Acad Sci USA 105:19915-19919

34. Bonner-Weir S, Li WC, Ouziel-Yahalom L, Guo L, Weir GC, Sharma A (2010) Beta-cell growth and regeneration: replication is only part of the story. Diabetes 59:2340-2348

35. Grant SF, Thorleifsson G, Reynisdottir I et al (2006) Variant of transcription factor 7-like 2 (TCF7L2) gene confers risk of type 2 diabetes. Nat Genet 38:320-323

36. Scott LJ, Bonnycastle LL, Willer CJ et al (2006) Association of transcription factor 7-like 2 (TCF7L2) variants with type 2 diabetes in a Finnish sample. Diabetes 55:2649-2653

37. Saxena R, Gianniny L, Burtt NP et al (2006) Common single nucleotide polymorphisms in TCF7L2 are reproducibly associated with type 2 diabetes and reduce the insulin response to glucose in nondiabetic individuals. Diabetes 55:2890-2895

38. Schafer SA, Tschritter O, Machicao F et al (2007) Impaired glucagon-like peptide-1-induced insulin secretion in carriers of transcription factor 7-like 2 (TCF7L2) gene polymorphisms. Diabetologia 50:2443-2450

39. Lyssenko V, Lupi R, Marchetti P et al (2007) Mechanisms by which common variants in the TCF7L2 gene increase risk of type 2 diabetes. J Clin Invest 117:2155-2163

40. Loder MK, da Silva XG, McDonald A, Rutter GA (2008) TCF7L2 controls insulin gene expression and insulin secretion in mature pancreatic beta-cells. Biochem Soc Trans 36:357-359

41. da Silva XG, Loder MK, McDonald A et al (2009) TCF7L2 regulates late events in insulin secretion from pancreatic islet betacells. Diabetes 58:894-905 